\title{
Study on lumbosacral nerve root compression using DTI
}

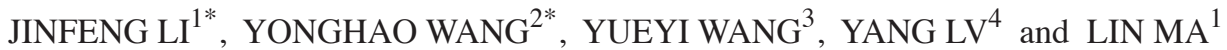 \\ ${ }^{1}$ Department of Radiology, Chinese People Liberation Army (PLA) General Hospital, Beijing 100853; \\ ${ }^{2}$ Department of Ultrasound, The 309th Hospital of Chinese PLA, Beijing 100091; ${ }^{3}$ Department of \\ Neonatology, Maternal and Child Care Center of Qianhuangdao, Qianhuangdao 066000; \\ ${ }^{4}$ Department of Nephrology, Chinese PLA General Hospital, Beijing 100853, P.R. China
}

Received February 11, 2016; Accepted May 16, 2016

DOI: 10.3892/br.2016.734

\begin{abstract}
Diffusion tensor imaging (DTI) can objectively describe the distribution of nerve roots in morphology, and provide a set of objective reference data on the quantitative indicators. The present study aimed to investigate the value of DTI in lumbosacral nerve root compression in patients with lumbar intervertebral disc degeneration. DTI was performed in 45 patients with lumbar intervertebral disc degeneration. Apparent diffusion coefficient (ADC) and fractional anisotropy (FA) values were measured in compressed and normal nerve roots. Fiber tracking imaging was also applied to observe the lumbosacral nerve roots. ADC value was significantly lower in the compressed group $\left(1.314 \pm 0.14 \mathrm{~mm}^{2} / \mathrm{sec}\right)$ compared to in the uncompressed group $\left(1.794 \pm 0.11 \mathrm{~mm}^{2} / \mathrm{sec}\right)(\mathrm{P}<0.05)$. The FA value was significantly lower in the compressed group $(0.196 \pm 0.020)$ compared to the uncompressed group $(0.272 \pm 0.016) \quad(\mathrm{P}<0.05)$. DTI can evidently reveal the compressed nerve roots. DTI could be used to evaluate the lumbosacral nerve injury in patients with lumbar intervertebral disc degeneration to quantitatively assess nerve roots.
\end{abstract}

\section{Introduction}

Magnetic resonance imaging (MRI) is a commonly used advanced inspection technique in clinical and scientific research. At present, the new technology mainly includes functional MRI, magnetic resonance spectroscopy, three-dimensional spoiled gradient recalled echo and diffusion tensor imaging (DTI). These technologies have been extensively applied in the brain, spinal cord lesions and tumors.

Correspondence to: Dr Lin Ma, Department of Radiology, Chinese People Liberation Army (PLA) General Hospital, 28 Fuxing Road, Beijing 100853, P.R. China

E-mail: drlinma@126.com

\section{${ }^{*}$ Contributed equally}

Key words: lumbar intervertebral disc, degeneration, diffusion tensor imaging, apparent diffusion coefficient, fractional anisotropy
DTI is an MR technique developed on the basis of the diffusion-weighted imaging (DWI). DTI collects attenuated signal intensity induced by a diffusion of water molecules in all directions of space through a diffusion sensitive gradient in numerous directions, quantitatively describes the three-dimensional trajectory of spatial diffusion, and can provide a diffusion direction characteristic of living tissue (1). Under physiological conditions, the diffusion rate of water molecules in each direction is different, i.e., diffusion anisotropy. DTI can measure the direction and degree of diffusion of the water molecules in a three-dimensional space, describe anisotropic characteristics of tissue and precisely investigate the distribution of fibers (2,3). Diffusion anisotropy has been found in muscle, and has also been detected in the spinal cord and white matter of living tissue in the late 1980s. White matter anisotropy is induced by paralleled distributed myelinated nerve fibers (4). The diffusion of white matter was faster in paralleled fibers compared to vertical fibers. This feature with a color mark can reflect spatial directivity of the white matter. Thus, the fastest diffusion direction is the direction of fiber distribution. It is the same in DTI of skeletal muscle and myocardium. With the advancement of studies regarding DTI, the DTI application has become an area of interest for human functional imaging. Since Clark et al (5) applied DWI in the spinal cord in 1999, the application of DTI in the spinal cord already has certain initial experience from ex vivo; in vivo animal to human. DTI is sensitive to the appearance of disease, but currently, studies on spinal cord DTI are scarce $(6,7)$. This is mainly due to the special structure of the spinal cord. The cross-sectional area of the spinal cord is relatively small. The axial spinal cord is oval and uneven in thickness, so the spinal cord DTI requires an imaging sequence of high resolution and high signal-to-noise ratio.

DTI is the only method to non-invasively visually reveal fiber tractography, a unique method to evaluate pathophysiology of spinal cord diseases, and is mainly used to diagnose and assess injury, cancer, inflammation and myelomalacia. DTI is not only extensively applied in the diagnosis and evaluation of spinal cord diseases, but also in other diseases. The fibrous ring of the intervertebral disc is composed of arranged collagen fibers, and has directivity. Therefore, DTI can exhibit the distribution of fibers of the fibrous ring in vivo. As the only method to exhibit a nerve fiber bundle in vivo, the application 
of DTI in the central nervous system is becoming increasingly extensive.

\section{Materials and methods}

Subjects. A total of 45 subjects with intervertebral disc degeneration, at the age of 23-66 years (average, $55.98 \pm 11.98$ years), were enrolled in the study, including 25 males and 20 females. All the subjects suffered from varying degrees of intervertebral disc degeneration and spinal canal stenosis. Tumor and various metabolic diseases were excluded. All the subjects signed informed consent. The study was approved by the Ethics Committee of the Chinese People Liberation Army (PLA) General Hospital (Beijing, China).

Equipment. A 3.0 T superconductive MRI machine (MR750; GE Healthcare, Milwaukee, WI, USA) was used with an 8 -channel spine coil, gradient field $40 \mathrm{mT} / \mathrm{m}$ and gradient switching rate $150 \mathrm{mT} / \mathrm{m} \cdot \mathrm{sec}$.

Image collection. An axial DTI scan was conducted with a single-shot spin-echo echo planar imaging sequence with the following parameters: TR/TE $=10,000 / 72.3 \mathrm{msec} ; \mathrm{b}$ value, $600 \mathrm{sec} / \mathrm{mm}^{2}$; slice thickness, $3.0 \mathrm{~mm}$; layer spacing, 0 ; number of layers, 39; matrix, 128x128; FOV, 24x24 cm; and NEX 1.0, diffusion sensitive gradient direction 15 .

Experimental design and implementation. According to the nerve root compression conditions, samples were divided into the compressed and uncompressed groups. The scan was performed by a technician from the Department of Radiology (Chinese PLA General Hospital). Prior to scanning, subjects were informed that their body would remain stationary during the scan.

Image analysis. Original images of DTI were loaded onto the AW4.4 (Advantage Windows; GE Healthcare) workstation, and measured using FuncTool software. The apparent diffusion coefficient (ADC) and fractional anisotropy (FA) were calculated. The region of interest (ROI) was placed on three continuous scan planes, once every plane. Nerve roots at the proximal, central and distal intervertebral foramina were separately measured (Fig. 1) three times. The average value of the three measured results was considered the final ADC and FA values. The ROI size was $20-40 \mathrm{~mm}^{2}$ (Fig. 1). ADC and FA values of the bilateral nerve roots in $\mathrm{L}_{3}-\mathrm{L}_{4}, \mathrm{~L}_{4}-\mathrm{L}_{5}$ and $\mathrm{L}_{5}-\mathrm{S}_{1}$ were measured in 45 subjects.

Statistical analysis. Quantitative data are expressed as mean \pm standard deviation, and analyzed using a group t-test between the two groups. $\mathrm{P}<0.05$ was considered to indicate a statistically significant difference.

\section{Results}

Comparison of $A D C$ and $F A$ values in the compressed and uncompressed groups. Among the 45 subjects, the number of nerve roots was 122 in the compressed group and 148 in the uncompressed group. Group t-test indicated that the $\mathrm{ADC}$ values were significantly lower in the compressed

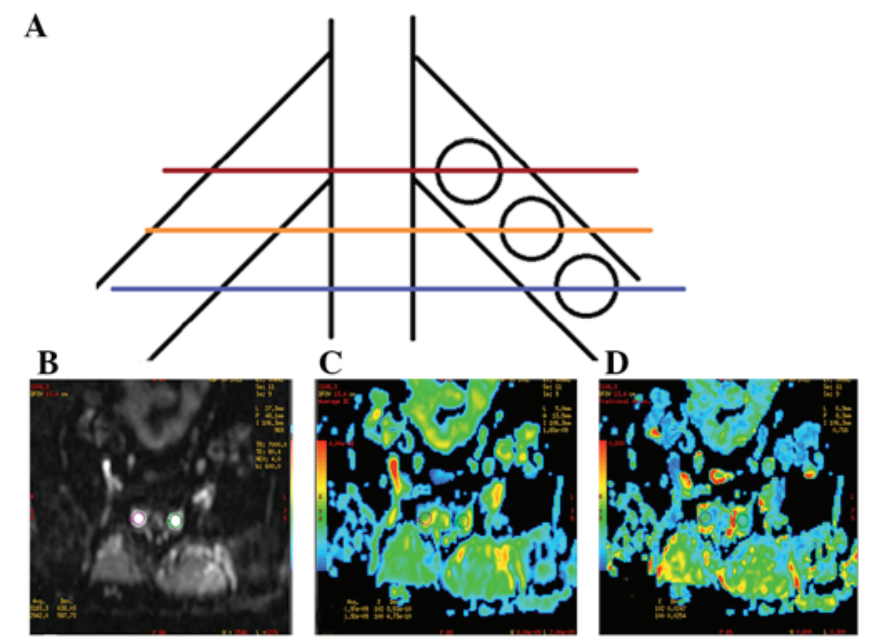

Figure 1. (A) Schematic diagram of a measuring plane. The red, yellow and blue lines are the continuous scan planes at the proximal, central and distal intervertebral foramina, respectively. (B-D) Similar round region of interest was placed at the nerve root of the proximal intervertebral foramina to measure ADC and FA values, as far as possible to avoid the surrounding cerebrospinal fluid. (B) $\mathrm{b}=0 \mathrm{sec} / \mathrm{mm}^{2}$, (C) ADC image and (D) FA image. ADC, apparent diffusion coefficient; FA, fractional anisotropy.

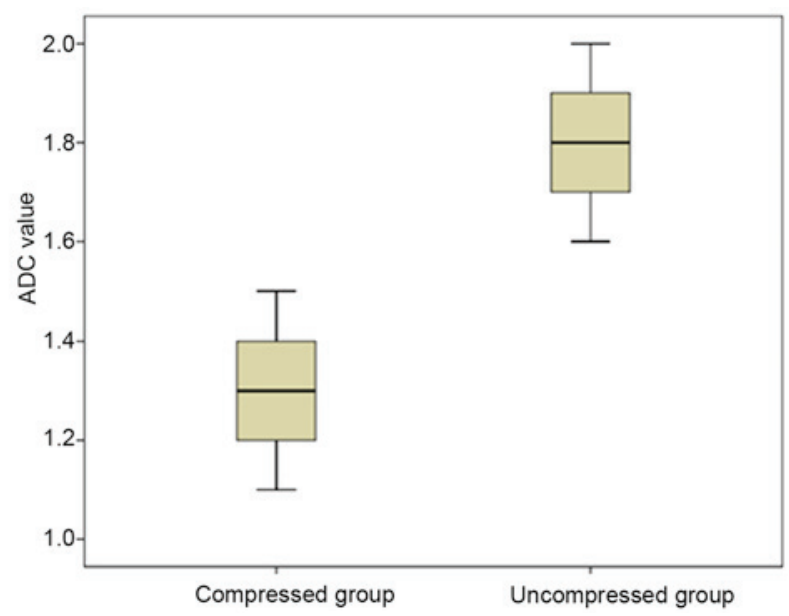

Figure 2. ADC values of the lumbosacral nerve roots. The ADC values of the nerve roots were evidently lower in the compressed group compared to the uncompressed group $(\mathrm{t}=31.314 ; \mathrm{P}=0.001)$. ADC, apparent diffusion coefficient.

group $\left(1.314 \pm 0.140 \times 10^{-3} \mathrm{~mm}^{2} / \mathrm{sec}\right)$ compared to the uncompressed group $\left(1.794 \pm 0.111 \mathrm{~mm}^{2} / \mathrm{sec}\right)(\mathrm{P}=0.001)$ (Fig. 2). FA values were significantly lower in the compressed group $(0.196 \pm 0.020)$ compared to the uncompressed group $(0.272 \pm 0.016)(\mathrm{P}=0.013$; Fig. 3$)$.

Tracer display. Among the 45 subjects, the DTI scan exhibited the distribution and compression of the nerve root in 36 subjects (Fig. 4).

\section{Discussion}

The spinal cord reaches the lumbar spinal canal, and ends at the corresponding level of the $\mathrm{L}_{1}-\mathrm{L}_{2}$ intervertebral disc. The lumbar, sacral and caudal nerve roots of the ventral and dorsal 


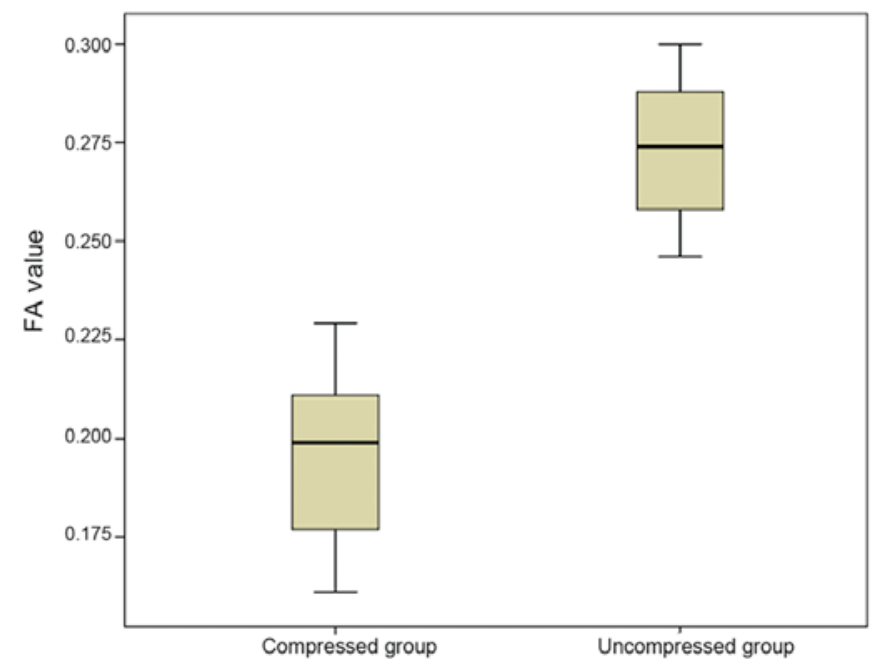

Figure 3. FA values of the lumbosacral nerve roots. The FA values of the nerve roots were evidently lower in the compressed group compared to the uncompressed group $(\mathrm{t}=34.521 ; \mathrm{P}=0.013)$. FA, fractional anisotropy.

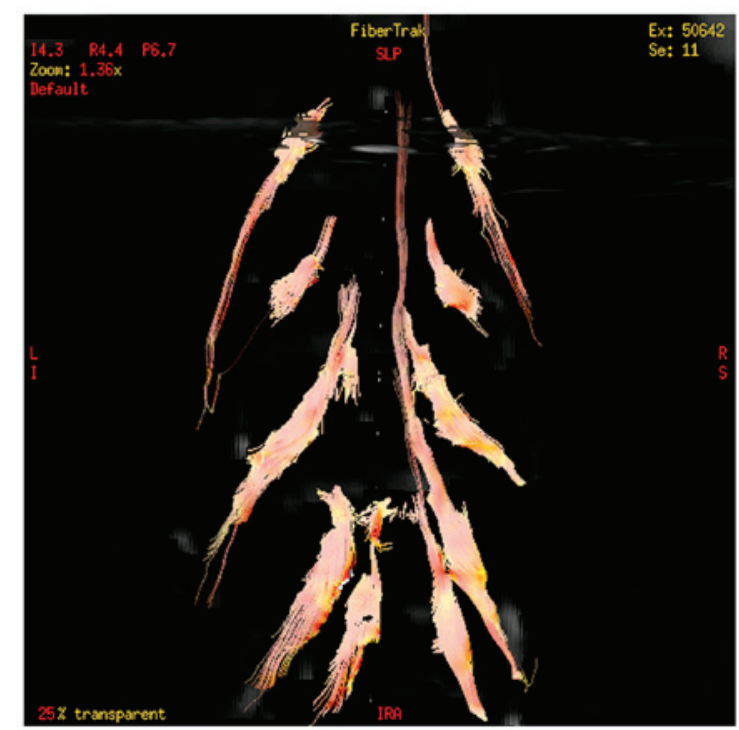

Figure 4. Apparent compression of the left $\mathrm{L}_{5}$ nerve roots revealed by diffusion tensor imaging tractography.

sections fix the spinal cord in the spinal canal. Descending nerve roots from the spinal cord go sequentially into their respective intervertebral foramina. Local nerve root morphology is similar to horsetail fibers, and is therefore known as cauda equina. Certain degenerative changes, such as intervertebral disc protrusion and the thickening of ligamentum flavum, can impact nerve roots and cause pain. Nucleus pulposus of the prolapsed intervertebral disc can produce an inflammatory mediator, impact the nerve root, and lead to pain.

With the rapid development of MRI and the increasing maturity of image processing technologies, numerous processing technologies of computational neuroanatomy are emerging. DTI can objectively describe the distribution of nerve roots in morphology, and provide a set of objective reference data on the quantitative indicators. A previous study verified that the effects of age and gender on ADC and FA values were not apparent in normal nerve roots (1). DTI, as a new imaging technique, can sensitively find the nerve root changes by measuring the ADC and FA values. Using the conventional method on ROI, according to the size of the nerve root cross-section at the proximal, central and distal intervertebral foramina, ROI was generally set at $30-70 \mathrm{~mm}^{2}$, and the effect of ROI size on measurement results was not evident (1).

DTI is a new and non-invasive MRI technique, and is mainly used to quantitatively evaluate microscopic structural changes of the nervous system, and to detect the blocking effect of cell membranes and myelin sheath on the movement of water molecules in the brain, particularly diffusion of water molecules in fiber bundles of the white matter, such as high anisotropy $(2,3)$. Thus, the diffusion was faster in water molecules parallel to the direction of axon and myelin sheath than in vertical direction $(2,3)$. The structure of fiber bundles observed by FA images was basically identical to that of the real fiber bundles (4). The voxel analysis of FA value can sensitively reflect brain lesions to a certain degree (6,7). The structure of fiber bundles of lumbosacral nerve roots is identical to that of intracranial nerve fibers, so their imaging principles are identical.

The imaging method of lumbosacral nerve roots consists of 3D FIESTA, MR PROSET technology, MRSSI (selective excitation) technology CUBE-FLEX and DTI. Several previous methods involve three-dimensional volumetric imaging. Occasionally, tissues with similar signals may interfere with the image quality. For example, vascular effects may interfere with nerve root display. DTI of lumbosacral nerve roots has been initially used in clinical research, but has not been extensively employed in the clinic due to the limitation of computer processing and imaging results. This method can provide a quantitative reference value of nerve root (ADC and FA values). In the reconstruction of the fiber bundle, a desired minimum threshold should be determined. If the minimum threshold is set too low, the reconstructed nerve fibers contain too many tissues and the edge is rough, so therefore the form of normal nerve fibers will be lost. If the minimum threshold is set too high, nerve fibers are rare. In the present study, the minimum threshold was set at $\sim 200$, the obtained DTI images can objectively reflect the real condition of nerve roots. The biggest advantage of DTI is to quantify the research index. The present results revealed that the ADC and FA values of the compressed nerve roots were evidently lower compared to that of the uncompressed nerve roots, which is consistent with a previous study (1).

In conclusion, the ADC and FA values of the nerve roots were evidently lower in the compressed group compared to the uncompressed group. DTI tractography can satisfactorily exhibit the compression of nerve roots and can quantitatively assess the compression of lumbosacral nerve roots in patients with lumbar disc degeneration.

\section{Acknowledgements}

The present study was supported by the PLA 12th five-year research project (grant no. CWS11J101).

\section{References}

1. Kitamura M, Eguchi Y, Inoue G, Orita S, Takaso M, Ochiai N, Kishida S, Kuniyoshi K, Aoki Y, Nakamura J, et al: A case of symptomatic extra-foraminal lumbosacral stenosis ('far-out syndrome') diagnosed by diffusion tensor imaging. Spine 37: E854-E857, 2012. 
2. Basser PJ: Inferring microstructural features and the physiological state of tissues from diffusion-weighted images. NMR Biomed 8: 333-344, 1995

3. Basser PJ, Mattiello J and LeBihan D: MR diffusion tensor spectroscopy and imaging. Biophys J 66: 259-267, 1994.

4. Pierpaoli C, Jezzard P, Basser PJ, Barnett A and Di Chiro G: Diffusion tensor MR imaging of the human brain. Radiology 201: 637-648, 1996

5. Clark CA, Barker GJ and Tofts PS: Magnetic resonance diffusion imaging of the human cervical spinal cord in vivo. Magn Reson Med 41: 1269-1273, 1999.
6. Albrecht J, Dellani PR, Müller MJ, Schermuly I, Beck M, Stoeter P, Gerhard A and Fellgiebel A: Voxel based analyses of diffusion tensor imaging in Fabry disease. J Neurol Neurosurg Psychiatry 78: 964-969, 2007.

7. Abe O, Yamasue H, Kasai K, Yamada H, Aoki S, Iwanami A, Ohtani T, Masutani Y, Kato N and Ohtomo K: Voxel-based diffusion tensor analysis reveals aberrant anterior cingulum integrity in posttraumatic stress disorder due to terrorism. Psychiatry Res 146: 231-242, 2006. 